shows perceptible opalescence with silver nitrate, while the other does not. But if, however, the washing of the silica is carried on for a long time, even after the $P_{H}$ of the wash water has become equal to that of the water used, the potassium nitrate solution fails to show any trace of a chloride.

Regarding the statement in par. 4 of his letter, I think Dr. Joseph is scarcely doing me justice in suggesting that these obvious precautions were not taken. The silica or the solutions never came in contact with filter papers. Transparent fused silica vessels were mostly used. Where glass vessels were used (resistance glass) it was ascertained that the results were not due to a reaction with the vessel We have several times repeated and confirmed our observations.

Dr. Joseph says nothing about the increase in the negative charge of the surface at low concentrations of neutral salts, which to my mind definitely proves the primary adsorption of anions, and consequently of electrolytes including acids which contain these anions.

Similar experiments have been done with manganese dioxide, corroborating our point of view. We have communicated a paper to the Journal of the Indian Chemical Society, "On the Nature of Hydrolytic Adsorption, Pt. I.," in which the theoretical considerations advanced in previous papers have been developed, and a brief summary of the results obtained in this laboratory during the last four years has also been given. Full experimental results will be published later.

I would take this opportunity to point out that we distinguish between three types of adsorption: (I) primary adsorption, resulting from the chemical affinity of the atoms on the surface (cf. Langmuir), (2) electrical adsorption, and (3) adsorption of solutes (or rather solutions) resulting from the capacity of the substance to adsorb water. The adsorption referred to in my letter in NATURE of April 4 (p. 497) is of the last-mentioned type, and is different from the small amounts of primary adsorption referred to in my letter of January $3 \mathrm{I}$. Physical Chemistry Laboratory,

University College of Science and Technology, Calcutta.

\section{Gas-pressure, Radiation-pressure, and Entropy in the Interior of a Star.}

IN the simplest form of Eddington's theory of stellar constitution (vide papers in Mon. Not. R.A.S. ; and Astrophysical Journal, 48, 205, I918), the assumption is made that a certain quantity, $\beta$, is constant throughout a given star; $\beta$ turns out to be the ratio of the gas-pressure to the total pressure (which is the sum of the gas-pressure and the radiation-pressure). The present note shows that the second law of thermodynamics provides a theoretical background for this assumption of Eddington's-justification of which has been largely empirical heretofore.

Write $S$ for the entropy per unit volume at any point within a star, $n$ for the number of molecules per unit volume, $d V$ for a volume-element, and $V$ for the total volume of the star. A feature of Eddington's method is that the volume, $V$, and the total number of molecules, $\int n d V$ (total mass divided by molecular weight), are supposed-at a certain point in the solution-to be given. Now $S$ may be taken as a function of the two independent variables, $V$ and $n$. Suppose that the distribution of matter within the star is infinitesimally varied, subject to the two restrictions mentioned; so that

$\delta V=\delta j d V=0$
$\int \delta n d V=0$,

and where the integrations are performed throughout the
entire volume of the star. The condition for stable equilibrium is that the total entropy is a maximum, or

$$
\delta \int S d V=0 .
$$

In consequence of $(\mathrm{I})$, this is equivalent to

$$
\int \frac{c S}{i n} i n d V=0 . \quad \text {. . . . }
$$

Comparison of (4) and.(2) necessitates that

$$
\frac{\hat{c} S}{\partial n}=b, \quad . \quad . \quad . \quad \text {. }
$$

a constant throughout $V$.

It remains to express the relation between $b$ and $\beta$. Taking the entropy as that of the radiation alone,

$$
S=\frac{4}{3} a T^{3}=\frac{4 p^{\prime}}{T}
$$

where $T$ is the absolute temperature of the elemental region; $a T^{4}$ the radiant energy per unit volume; and $p^{\prime}$ the radiation-pressure. Since the gas-pressure, $p$, is given by $n R T$ (where $R$ is the gas-constant per molecule),

Comparing with (5),

$$
S=4 n R p^{\prime} \mid p .
$$

$$
b=\frac{4 R p^{\prime}}{p}=4 R \frac{I-\beta}{\beta} ;
$$

and $\beta$ is constant throughout the star.

This treatment neglects the entropy of the gas. Furthermore, owing to varying ionisation with the varying distributions of matter, (2) above is only an approximation. Consequently the way is left open for more refined theories, according to which $\beta$ and $b$ may not be constant throughout a given star.

Princeton University Observatory, $Q$. S'TEWART. July 28.

\section{The Experimental Transmission of Cutaneous Leishmaniasis to Man from Phlebotomus papatasii.}

Sergent, Ed. et Et., Parrot, L., Donatien, H., and Béguet, M. (C.R. Acad. Sci., vol. I73, No. 2I, pp. I030-I032), first successfully transmitted cutaneous Leishmaniasis to man from sandflies. Their experiment consisted of dividing a batch of 559 sandflies into 23 batches, crushing the sandflies in saline, and inoculating scarified points on the arms of volunteers. The sandflies were caught in Biskra, an endemic centre of cutaneous Leishmaniasis, and the experiment was performed in Algiers, where locally acquired cases of the disease are unknown. One experiment (from a batch of 7 specimens of Phlebotomus papatasii) was successful, Leishman-Donovan bodies being found from a papule which appeared 2 months and 24 days after the experiment.

A batch of I98 sandflies (I9I Phlebotomus papatasii, I 6 of and 175 o $q, 7 P$. minutus, I $\delta$ and $6 \% Q$ ) were caught in Jericho on June 25 last, and brought to Jerusalem for dissection. On June 26 a female Phlebotomus papatasii was found heavily infected with Herpetomonas, flagellates being present in the œsophagus, œsophageal diverticulum, midgut, and hindgut. The alimentary tract contained no trace of blood and the abdomen was full of ripe or almost ripe eggs. A large number of the flagellates were found attached to the under surface of the osophageal

NO. 2913 , VOL. I I 6] 